\title{
Comparative safety of anesthetic type for hip fracture surgery in adults: retrospective cohort study
}

\author{
(C) $(1) \Theta$ OPEN ACCESS
}

\author{
Elisabetta Patorno instructor ${ }^{1}$, Mark D Neuman assistant professor ${ }^{2}$, Sebastian Schneeweiss \\ professor $^{1}$, Helen Mogun programmer ${ }^{1}$, Brian T Bateman assistant professor ${ }^{13}$
}

'Division of Pharmacoepidemiology and Pharmacoeconomics, Department of Medicine, Brigham and Women's Hospital, Harvard Medical School, Boston, MA 02120, USA; ${ }^{2}$ Department of Anesthesiology and Critical Care, Perelman School of Medicine, University of Pennsylvania, Philadelphia, PA, USA; ${ }^{3}$ Department of Anesthesia, Critical Care, and Pain Medicine, Massachusetts General Hospital, Boston, MA, USA

\begin{abstract}
Objective To evaluate the effect of anesthesia type on the risk of in-hospital mortality among adults undergoing hip fracture surgery in the United States.

Design Retrospective cohort study.

Setting Premier research database, United States.

Participants 73284 adults undergoing hip fracture surgery on hospital day 2 or greater between 2007 and 2011. Of those, 61554 (84.0\%) received general anesthesia, 6939 (9.5\%) regional anesthesia, and 4791 $(6.5 \%)$ combined general and regional anesthesia.
\end{abstract}

Main outcome measure In-hospital all cause mortality.

Results In-hospital deaths occurred in 1362 (2.2\%) patients receiving general anesthesia, $144(2.1 \%)$ receiving regional anesthesia, and 115 (2.4\%) receiving combined anesthesia. In the multivariable adjusted analysis, when compared with general anesthesia the mortality risk did not differ significantly between regional anesthesia (risk ratio $0.93,95 \%$ confidence interval 0.78 to 1.11 ) or combined anesthesia $(1.00,0.82$ to 1.22). A mixed effects analysis accounting for differences between hospitals produced similar results: compared with general anesthesia the risk from regional anesthesia was 0.91 (0.75 to 1.10) and from combined anesthesia was 0.98 (0.79 to 1.21$)$. Findings were also consistent in subgroup analyses.

Conclusions In this large nationwide sample of hospital admissions, mortality risk did not differ significantly by anesthesia type among patients undergoing hip fracture surgery. Our results suggest that if the previously posited beneficial effect of regional anesthesia on short term mortality exists, it is likely to be more modest than previously reported.

\section{Introduction}

Hip fractures are serious injuries, with an estimated annual incidence of more than 1.5 million worldwide. ${ }^{1}$ Owing to the global increase of the population aged 65 years and over, the total number of hip fractures is expected to surpass six million by $2050 .^{2}$ In 2010 in the United States, there were 303000 hospital admissions for hip fractures among adults. ${ }^{3} \mathrm{Hip}$ fractures are associated with a substantial risk of morbidity and mortality, with approximately $1-6 \%$ of patients dying during hospital stay ${ }^{4-6}$ and 4-10\% dying within 30 days of admission, ${ }^{4-8}$ largely due to high rates of pulmonary and cardiovascular complications. ${ }^{8}$ Most hip fractures are treated with surgical repair, which represents one of the most common orthopedic procedures. ${ }^{10}$ Few interventions have been shown to decrease mortality in patients with hip fracture. ${ }^{11} 12$

Anesthesia type has been hypothesized to have an effect on mortality among patients undergoing a range of surgical procedures. Specific advantages associated with the different anesthetic techniques may play a part in this effect. Regional anesthesia leads to avoidance of airway management, decreased blood loss, potentially reduced risk of deep venous thrombosis, and improved postoperative analgesia. Conversely, general anesthesia may be associated with a more stable hemodynamic state than regional anesthesia. ${ }^{10} 1314$

Several investigations have attempted to determine whether regional anesthesia offers benefits over general anesthesia for surgeries generally, but evidence remains conflicting. In 2000 a meta-analyses of 141 clinical trials showed a reduction in postoperative mortality associated with regional anesthesia, with the subsequent recommendation for more widespread use of neuraxial blockade. ${ }^{15}$ However, most of the studies included in this meta-analysis were small ( $<100$ patients); were dated, with many trials published in the 1980s; and often did not distinguish between regional and combined general and regional anesthesia. ${ }^{16}$ More recent meta-analyses of randomized clinical 
studies have produced mixed results and have not been able to conclude if anesthetic type influences mortality. ${ }^{17-20}$

For hip fracture surgery, two meta-analyses of 15 and 22 randomized clinical trials comparing regional with general anesthesia found borderline significant results of a reduced short term risk of mortality associated with regional anesthesia, but no evidence of a decreased risk at three months post-surgery or beyond ${ }^{1021}$; all included studies had important methodological flaws. A review of 34 clinical trials concluded that the limited evidence available does not permit a definitive conclusion to be drawn on type of anesthesia and mortality risk in patients undergoing hip fracture surgery. ${ }^{22} \mathrm{~A}$ few large observational studies have been performed to assess the impact of anesthesia type on mortality among such patients, with discordant results. While some of these studies found that the use of regional anesthesia was associated with a decreased risk of mortality, ${ }^{56}$ others found no difference in outcomes. ${ }^{43}$ We evaluated the effect of anesthesia type on the risk of in-hospital mortality among adults undergoing hip fracture surgery using the largest nationwide database of inpatient hospital admissions, which contains information on types of anesthesia available in the United States.

\section{Methods}

\section{Data source}

We collected data from the Premier research database, which contains data on healthcare utilization by inpatients from the records of about one sixth of all hospital admissions in the United States. Premier is a national organization focused on healthcare performance improvement that provides data services to hospitals, including tabulation and benchmarking against the performance of other institutions, and its database records information on all inpatients from approximately 500 hospitals across the United States (numbers vary slightly by year). Data are collected from member hospitals through Premier's informatics products, and Premier then provides information to the hospitals for benchmarking purposes. All patients treated at these hospitals are included in the database, independent of payer status (Medicaid, Medicare, or commercial insurance). The hospitals are primarily non-profit, non-governmental, community and teaching hospitals.

The database also includes date stamped charges for all drugs, procedures, and diagnostic tests during inpatient admissions (charge codes). Patients' personal and hospital characteristics, discharge diagnoses, and discharge status are also recorded. The database reflects all the information that is captured for billing purposes at the hospital level; thus data are expected to be accurate. Data are also routinely audited, verified, and validated to ensure that the use of supplies and other hospital resources are within an acceptable range, but Premier does not directly verify that submitted data match individual patients' medical records.$^{24}$ Preliminary analysis done by the Premier organization, comparing characteristics of patients and hospitals for Premier hospitals using the Premier research database with those from the national hospital discharge survey suggests that the profile of patients treated at hospitals participating in the Premier research database is similar to those treated nationally (statement of work document, Premier, 2012). Several studies have used Premier data to study perioperative drug use and health outcomes. ${ }^{24-27}$

\section{Study population}

The study population included adults aged 18 years and older who underwent hip fracture surgery between 1 October 2007 and 30 September 2011.We identified surgical repair of hip fracture based on a previous definition. ${ }^{6} \mathrm{We}$ selected all discharges with a principal or a secondary diagnosis code for hip fracture, including disease related fractures of the femoral neck (international classification of disease, ninth revision, clinical modification ${ }^{28}$ diagnosis codes 820, 733.14); within this group we restricted to patients with a procedure code corresponding to open reduction, internal fixation, hemiarthroplasty, or total hip arthroplasty (ICD-9-CM codes $00.7,79.15,79.25,79.35,81.40,81.51-3)$. We excluded patients undergoing closed reduction without internal fixation (79.05) and those with an ICD-9-CM code suggestive of multiple trauma, including other fractures (800-819, 821-829), dislocations (830-839), sprains (840-848), injuries (850-854, 860-887, 925-929), and accidents (E800-E807, E810-E838, E840-E848). For the main analysis, we included only those patients who underwent surgical repair of hip fracture on the second day of hospital admission or thereafter. We did this to allow for a baseline period in which information on patients' comorbidities and other risk factors was collected; these factors may affect both the choice of anesthesia type and the risk of mortality and thus act as confounders.

\section{Exposure and study outcome}

We defined exposure to a specific anesthesia type by one or more charge codes for general or regional anesthesia generated on the day of surgery. We classified patients as having received general anesthesia if they had charges for general anesthesia only, whereas we classified patients who had charges for epidural or spinal anesthesia as having received regional anesthesia. Study participants having charges for both general and regional anesthesia were classified as having received combined general and regional anesthesia. To limit the chances of misclassification for exposure, we excluded from the analysis those patients without a detailed and clear indication of anesthesia type on the day of surgery $(n=32149)$. These patients were not dissimilar from the other patients for characteristics and risk of in-hospital mortality (see supplementary tables A1 and A2). An equivalent extent of inconclusive information on anesthesia type has been previously observed with the Premier research database. ${ }^{29}$

The study outcome was in-hospital mortality as recorded in the database. We chose in-hospital mortality as the study endpoint because the immediate postoperative period is likely to be more causally related to the type of anesthesia used during surgery than in subsequent periods. In-hospital mortality was chosen as primary outcome in a recent US observational study, ${ }^{6}$ and a seven day mortality outcome has been recognized as more likely to reflect anesthetic related complications than longer follow-up periods. ${ }^{4}$

\section{Patient and hospital level characteristics}

We identified the following patient and hospital level characteristics as potential confounders: personal characteristics, hospitalization and surgery characteristics, fracture type, chronic comorbid conditions, inpatient use of drugs and services before the day of surgery, and hospital characteristics. Personal characteristics included age on admission, sex, race or ethnicity (white, black, hispanic, or other), and marital status (married, single, or other). Hospitalization and surgery characteristics included type of admission (emergency room, non-healthcare facility, or other), year of admission, number of days the patient was in hospital before surgery, and type of surgical procedure (closed or open reduction of fracture, total or partial hip replacement, other hip procedures). Using discharge diagnoses 
we identified fracture type (transcervical, intertrochanteric, subtrochanteric, other trochanteric fractures, fractures of unspecified part of neck of femur, disease related fractures of neck of femur) and presence of chronic comorbid conditions. Chronic comorbid conditions included chronic hypertension, diabetes, hyperlipidemia, chronic obstructive pulmonary disease, previous myocardial infarction, history of percutaneous coronary procedure, other forms of ischemic heart disease, chronic heart failure, chronic cardiac dysrhythmia, previous stroke, renal disease, liver disease, chronic hemostatic disorders, dementia, cancer, osteoarthritis, rheumatoid arthritis, obesity, smoking (former or current), alcohol or drug misuse or dependence, and history of peptic ulcer disease. Although these conditions were recorded as discharge diagnoses, all these chronic conditions would have been present before admission. We also calculated the Romano modification of the Charlson comorbidity index for each patient by using conditions identified by discharge diagnoses. ${ }^{30}$ We evaluated charges for drugs and procedures before the day of surgery as markers of severity of comorbid illness. Charges for drug use included angiotensin converting enzyme inhibitors, angiotensin receptor blockers, $\beta$ blockers, calcium channel blockers, nitrates, thiazide diuretics, loop diuretics, aldosterone antagonists, digoxin, rhythm control drugs, oral antidiabetes drugs or insulin, treatment for chronic obstructive respiratory diseases (inhaled $\beta 2$ agonists, inhaled steroids, leukotriene inhibitors, ipratropium, or theophylline), statins, aspirin, other antiplatelets, warfarin, subcutaneous anticoagulants, intravenous heparin, and systemic corticosteroids. We also assessed charges for inpatient services before surgery, including preoperative admission to an intensive care unit, oxygen use, telemetry, echocardiography, dialysis, transfusion of packed red blood cells, and transfusion of fresh frozen plasma. Finally, we assessed the characteristics of the hospitals in which patients were treated and recorded whether they were teaching hospitals and whether they were located in an urban or rural location. We estimated the annualized volume of surgeries for hip fracture repair performed by each hospital by dividing the total number of such surgeries for each hospital during the study period by the number of years that each hospital performed one or more surgical repairs for hip fracture. We ranked hospitals in order of annualized volume and then categorized them into thirds of volume (high, medium, and low volume hospitals). ${ }^{31}$ We classified the region of the hospital as mid-west, north east, south, or west.

\section{Statistical analysis}

We cross tabulated patient characteristics by type of anesthesia. For each anesthesia type, we calculated number of patients, number of events, and the in-hospital mortality risk. We determined the unadjusted risk ratios with $95 \%$ confidence intervals for in-hospital mortality, comparing patients exposed to regional anesthesia and combined general and regional anesthesia with patients exposed to general anesthesia. Because of the low event rate, we report the odds ratios from logistic regression analyses as risk ratios. ${ }^{32}$

To control for confounding by indication, we fit a multivariable logistic regression model, from which we estimated a relative risk and 95\% confidence interval for in-hospital mortality. The model included all personal, hospital admission, and surgery characteristics, fracture types, chronic comorbid conditions, inpatient use of drugs and services before the day of surgery, and hospital characteristics, as defined previously.

To test the robustness of our primary findings we performed several sensitivity analyses. Firstly, we used mixed effects logistic regression models to compare anesthetic technique, adjusting for potential differences between hospitals. The hospital identifying variable was modeled as a normally distributed random intercept, and the patient and hospital level characteristics were modeled as fixed effects variables. This analysis provided information about the effect of anesthesia type on mortality risk after having adjusted for the individual hospital effect. Secondly, to test the generalizability of our findings, we reassessed the risk of in-hospital mortality after having extended the study cohort to include all patients undergoing hip fracture surgery independently of when the surgical procedure took place during the hospital admission, thus including also the patients who had surgery on the first day of hospital admission. Although important in establishing generalizability, estimates from these analyses are more likely to be biased than those obtained in the primary analysis owing to more limited ability to control for confounding (as less baseline information is available before surgery). For this analysis, the multivariable logistic regression model included only personal, admission to hospital, and surgery characteristics, fracture types, chronic comorbid conditions, and hospital characteristics. Thirdly, we tested the potential for effect modification in subgroup analyses restricted to patients 75 years and older and to patients with no recorded diagnosis of cancer at discharge.

Statistical analyses were performed using SAS version 9.3 (SAS Institute, Cary, NC).

\section{Results}

Overall, 73284 patients underwent surgery for hip fracture repair between 1 October 2007 and 30 September 2011 and had information on the type of anesthesia received. Of those, 61554 $(84.0 \%)$ received general anesthesia, $6939(9.5 \%)$ received regional anesthesia, and $4791(6.5 \%)$ received both general and regional anesthesia (figure $\Downarrow$ ). Hospital length of stay after surgery was equivalent across the three anesthetic groups (median five days), thus providing an equal opportunity to observe outcomes in all the groups. In total, 1621 (2.2\%) deaths occurred during hospital admission, which is consistent with previously reported findings. ${ }^{46}$

Baseline characteristics were generally similar across anesthetic groups (table $1 \Downarrow$ ), with a few differences noted. Compared with patients receiving other types of anesthesia, those receiving general anesthesia were younger, less likely to be women, more likely to be admitted from a non-healthcare facility, more likely to undergo closed or open fracture reduction, and less likely to undergo partial hip replacement; they had a lower frequency of transcervical fractures and more often had diabetes but less often had a history of chronic obstructive pulmonary disease or dementia. Patients receiving general anesthesia were more likely to have received treatment with $\beta$ blockers, antidiabetes drugs, and statins, to have been admitted to an intensive care unit, or to have had dialysis, and were less likely to have received supplemental oxygen before surgery. These patients were more often treated at teaching and urban hospitals and at hospitals in the north east and mid-west. Conversely, patients receiving regional anesthesia were older, more likely to be women, more likely to be admitted through the emergency department, less likely to have white or black ethnicity, and less likely to have chronic renal disease.

Overall, $1362(2.2 \%)$ in-hospital deaths occurred in patients receiving general anesthesia, $144(2.1 \%)$ in patients receiving regional anesthesia, and $115(2.4 \%)$ in patients receiving combined anesthesia (table $2 \Downarrow$ ). The unadjusted analyses showed no significant differences in the risk of in-hospital mortality 
across the anesthetic groups for patients receiving regional anesthesia compared with general anesthesia (risk ratio 0.94 , $95 \%$ confidence interval 0.79 to 1.11 ) or combined anesthesia compared with general anesthesia (1.09, 0.90 to 1.32$)$. Similarly, in the multivariable analysis there was no statistically significant difference in mortality risk associated with the use of either regional compared with general anesthesia ( $0.93,0.78$ to 1.11$)$ or combined compared with general anesthesia $(1.00,0.82$ to 1.22).

The mixed effects analysis, which accounted for differences between hospitals, produced results consistent with those of the main analysis (regional $v$ general anesthesia $0.91,0.75$ to 1.10 , and combined $v$ general anesthesia $0.98,0.79$ to 1.21 ). When the study population was extended to include those patients who had surgery on the first day of hospital admission, the results remained consistent (regional $v$ general anesthesia $0.91(0.77$ to 1.08 ), and combined $v$ general anesthesia 0.97 ( 0.82 to 1.17 , table $3 \Downarrow$ ). Similarly, restricting the study population to patients with no diagnosis of cancer at discharge or to patients 75 years and older yielded a similar effect for regional or combined anesthesia compared with general anesthesia (table 3).

\section{Discussion}

Using a cohort of 73284 adult who underwent surgery for hip fracture in a nationwide sample of inpatient admissions in the United States, we found no evidence of a significant difference in the risk of in-hospital mortality associated with type of anesthesia. This result was robust across several sensitivity and subgroup analyses. However, we observed a not statistically significant $7 \%$ to $9 \%$ reduction in mortality risk associated with the use of regional anesthesia alone compared with general anesthesia, which does not permit the ruling out of the possibility of a modest beneficial effect in the routine care of patients undergoing hip fracture surgery.

\section{Comparison with other studies}

Whether there is a beneficial effect on mortality associated with the use of regional anesthesia has been an area of considerable controversy. Previous meta-analyses of randomized clinical trials ${ }^{1021}$ found a non-significant or borderline significant effect of regional anesthesia in reducing short term mortality among patients undergoing hip fracture surgery; however, all included trials had important methodological flaws. Similarly, a retrospective cohort study of 9425 patients aged 60 years and older who underwent surgical repair of hip fracture at 20 US hospitals between 1983 and 1993 found no difference between general and regional anesthesia for seven or 30 day mortality risk. ${ }^{4}$ Conversely, two subsequent observational studies found that the use of regional anesthesia was associated with a substantial decreased risk of mortality: Redcliff and colleagues found that in a cohort of 5683 community dwelling male veterans undergoing hip fracture surgery between 1998 and 2003 , general anesthesia was associated with a $27 \%$ increased risk of 30 day mortality compared with regional anesthesia ${ }^{5}$; Neuman and colleagues found that regional anesthesia was associated with a $29 \%$ reduced risk of in-hospital mortality compared with general anesthesia among 18158 patients undergoing surgical repair of hip fracture at 126 hospitals in New York State between 2007 and 2008. ${ }^{6}$ More recently, a large observational study based on 57397 patients who underwent hip fracture surgery in the United Kingdom in 2012 and had available information on anesthesia type and vital status, found no difference in the age and American Society of Anesthesiologists physical status adjusted 30 day mortality risk between general and spinal anesthesia. ${ }^{23}$ The results from our observational study, which derive from a cohort that is substantially larger than in these previous studies (allowing for more precise estimates), which account for many more comorbidities and their proxies using granular data (allowing for superior confounding control), and which are based on anesthesia type defined by charge codes (which results in minimal misclassification of exposure), suggest that there is either little or no effect of regional anesthesia on the risk of mortality after hip fracture surgery. If a beneficial effect of regional anesthesia on short term mortality exists, it is likely to be more modest than previously reported.

\section{Strengths and limitations of this study}

Our study has several strengths. To our knowledge, this is the largest population based study to evaluate the effect of anesthesia type on the risk of mortality among patients undergoing hip fracture surgery in a routine care setting using data from a nationwide sample. Through the use of the Premier research database, we were able to access detailed inpatient information, including drug treatment, procedure utilization, and patients' diagnoses. We used multiple approaches in the design and analysis of the study to minimize the potential for residual confounding, including the restriction to patients who had hip fracture surgery on the second day of hospital admission or thereafter, to allow for a baseline period in which information on comorbidities and their proxies could be collected, and the use of mixed effects logistic analysis to account for potential differences associated with individual hospitals. We also investigated the potential for effect modification in subgroup analyses restricted to patients 75 years and older and to patients with no recorded diagnosis of cancer at discharge. Further, we capture the type of anesthesia based on charge codes, which should result in minimal misclassification of exposure.

Some limitations need to be considered. Although the Premier research database provides detailed inpatient information, certain relevant clinical details (for example, functional status before the injury, measures of frailty, pulmonary function, body mass index) might not have been completely captured, as either not fully or not directly measured in administrative data, and thus may lead to residual confounding. We adjusted for confounding by restricting the study population to the patients who had surgery on hospital day 2 or later to identify and adjust for all relevant confounders and their proxies that might be associated with the choice of anesthesia type, and by using mixed effects analysis to account for potential differences between hospitals that might confound the association between anesthesia type and in-hospital mortality. Despite these efforts, some residual confounding is possible; however, for there to be a substantially beneficial effect of regional anesthesia, the amount of residual confounding would need to be implausibly large given the detailed patient level data available in the Premier research database. An additional limitation is that about $30 \%$ of the patients undergoing hip fracture surgery had no conclusive information on the type of anesthesia through the database, as previously observed. ${ }^{29}$ To ensure a highly specific definition of anesthesia type, we excluded those patients. However, patient and hospital level characteristics as well as in-hospital mortality risk were not dissimilar between patients with and without conclusive information on anesthesia type (see supplementary tables A1 and A2), suggesting that their exclusion is unlikely to affect our findings. Although all fully adjusted models controlled for dementia, a further limitation is the absence of accurate information for possible patient delirium on admission, which can be associated with the choice of anesthesia type and 
the risk of mortality. This is because the Premier research database collects information on ICD-9 diagnosis codes only at discharge and therefore does not discriminate between delirium that occurred on admission or subsequent to the hip surgery procedure. Another potential limitation is that to measure and account for baseline clinical characteristics that might influence the choice of anesthesia type, we restricted our principal analysis to patients who had surgery on hospital day 2 or later, which may affect the generalizability of our results. To tackle this potential problem, in a sensitivity analysis we examined the risk of in-hospital mortality including patients who had surgery on any day during their hospital admission. All the analyses yielded consistent results, suggesting that our findings are generalizable to all patients undergoing hip fracture surgery. Finally, while our results suggest the use of regional anesthesia in the United States is less common than in Europe, ${ }^{23}$ this difference alone should not impact on the causal effect of anesthesia type on mortality and thus on the generalizability of our results to non-US settings.

\section{Conclusions}

The findings from this large nationwide investigation do not support the hypothesis of a substantially beneficial effect of regional anesthesia compared with general anesthesia on all cause mortality, but suggest that if a beneficial effect of regional anesthesia on postoperative mortality exists, it is likely to be more modest than previously reported. Additional research with studies powered to detect effect heterogeneity is needed to explore if specific population subgroups may meaningfully benefit from the use of regional anesthesia. Based on our data, the choice of anesthesia type should put emphasis on considerations other than differences in short term mortality.

Contributors: EP and BTB were involved in all parts of the study. HM was involved in data analysis and revising the manuscript. MDN and SS were involved in designing the study and revising the manuscript. All authors had full access to all of the data (including statistical reports and tables) in the study and can take responsibility for the integrity of the data and the accuracy of the data analysis. EP and BTB are the guarantors.

Funding: This study was supported by the Division of Pharmacoepidemiology and Pharmacoeconomics, Brigham and Women's Hospital and Harvard Medical School and by grants to MDN from the Foundation for Anesthesia Education and Research (Rochester, $\mathrm{MN}$ ) and the National Institute on Aging (K08AG043548) and to BTB from the Eunice Kennedy Shriver National Institute Of Child Health \& Human Development of the National Institutes of Health under Award No K08HD075831. The content is solely the responsibility of the authors and does not necessarily represent the official views of the National Institutes of Health.

Competing interests: All authors have completed the ICMJE uniform disclosure form at www.icmje.org/coi_disclosure.pdf (available on request from the corresponding author) and declare: SS is principal investigator of the Harvard-Brigham Drug Safety and Risk Management Research Center funded by the Food and Drug Administration. His work is partially funded by grants/contracts from the Patient-Centered Outcomes Research Institute, the FDA, and the National Heart, Lung, and Blood Institute; SS is consultant to WHISCON and LLC, and to Aetion, a software manufacturer of which he also owns shares, and he is principal investigator of investigator initiated grants to the Brigham and Women's Hospital from Novartis and Boehringer-Ingelheim unrelated to the topic of this study.

Ethical approval: This study was approved by the institutional review board (No 2011P002580) of the Brigham and Women's Hospital, Boston, $\mathrm{MA}$, and a data use agreement was in place.
Data sharing: No additional data available.

Transparency: EP and BTB affirm that the manuscript is an honest, accurate, and transparent account of the study being reported; that no important aspects of the study have been omitted; and that any discrepancies from the study as planned (and, if relevant, registered) have been explained.

Johnell O, Kanis JA. An estimate of the worldwide prevalence and disability associated with osteoporotic fractures. Osteoporos Int 2006;17:1726-33.

2 Cooper C, Campion G, Melton LJ III. Hip fractures in the elderly: a worldwide projection. Osteoporosis Int 1992;2:285-9.

3 National Hospital Discharge Survey (NHDS), National Center for Health Statistics. 2014 http://205.207.175.93/hdi/ReportFolders/ReportFolders.aspx?IF_ActivePath=P,18.

4 O'Hara DA, Duff A, Berlin JA, Poses RM, Lawrence VA, Huber EC, et al. The effect of anesthetic technique on postoperative outcomes in hip fracture repair. Anesthesiology 2000;92:947-57.

5 Radcliff TA, Henderson WG, Stoner TJ, Khuri SF, Dohm M, Hutt E. Patient risk factors, operative care, and outcomes among older community-dwelling male veterans with hip fracture. J Bone Joint Surg Am 2008;90:34-42.

6 Neuman MD, Silber JH, Elkassabany NM, Ludwig JM, Fleisher LA. Comparative effectiveness of regional versus general anesthesia for hip fracture surgery in adults. Anesthesiology 2012;117:72-92.

7 Parker M, Johansen A. Hip fracture. BMJ 2006;333:27-30.

8 Roche JJ, Wenn RT, Sahota O, Moran CG. Effect of comorbidities and postoperative complications on mortality after hip fracture in elderly people: prospective observational cohort study. BMJ 2005:331:1374

9 Lawrence VA, Hilsenbeck SG, Noveck H, Poses RM, Carson JL. Medical complications and outcomes after hip fracture repair. Arch Intern Med 2002;162:2053-7.

10 Parker MJ, Handoll HH, Griffiths R. Anaesthesia for hip fracture surgery in adults. Cochrane Database Syst Rev 2004;4:CD000521.

11 Beaupre LA, Jones CA, Saunders LD, Johnston DW, Buckingham J, Majumdar SR. Best practices for elderly hip fracture patients: a systematic overview of the evidence. $J$ Gen Intern Med 2005:20:1019-25.

12 Neuman MD, Archan S, Karlawish JH, Schwartz JS, Fleisher LA. The relationship between short-term mortality and quality of care for hip fracture: a meta-analysis of clinical pathways for hip fracture. J Am Geriatr Soc 2009;57:2046-54

13 Modig J, Borg T, Bagge L, Saldeen T. Role of extradural and of general anaesthesia in fibrinolysis and coagulation after total hip replacement. Br J Anaesth 1983;55:625.

14 Modig J. Regional anaesthesia and blood loss. Acta Anaesthiol Scand Suppl 1988:89:44-8.

15 Rodgers A, Walker N, Schug S, McKee A, Kehlet H, van Zundert A, et al. Reduction of postoperative mortality and morbidity with epidural or spinal anaesthesia: results from overview of randomised trials. $B M J$ 2000;321:1493.

16 Kettner SC, Willschke $\mathrm{H}$, Marhofer P. Does regional anaesthesia really improve outcome? Br J Anaesth 2011:107(S1):i90-5.

17 Werawatganon T, Charuluxanun S. Patient controlled intravenous opioid analgesia versus continuous epidural analgesia for pain after intra-abdominal surgery. Cochrane Database Syst Rev 2005;1:CD004088.

18 Macfarlane AJR, Prasad GA, Chan VWS, Brull R. Does regional anesthesia improve outcome after total knee arthroplasty? Clin Orthop Relat Res 2009;467:2379-402.

19 Svircevic V, van Dijk D, Nierich AP, Passier MP, Kalkman CJ, van der Heijden GJ, et al. Meta-analysis of thoracic epidural anesthesia versus general anesthesia for cardiac surgery. Anesthesiology 2011;114:271-82.

20 Nishimori M, Ballantyne JC, Low JH. Epidural pain relief versus systemic opioid-based pain relief for abdominal aortic surgery. Cochrane Database Syst Rev 2006;3:CD005059.

21 Urwin SC, Parker MJ, Griffiths R. General versus regional anaesthesia for hip fracture surgery: a meta-analysis of randomized trials. Br J Anaesth 200084:450-5.

22 Luger TJ, Kammerlander C, Gosch M, Luger MF, Kammerlander-Knauer U, Roth T, et al. Neuroaxial versus general anaesthesia in geriatric patients for hip fracture surgery: does it matter? Osteoporos Int 2010;21(Suppl 4):S555-72.

23 White SM, Moppett IK, Griffiths R. Outcome by mode of anaesthesia for hip fracture surgery. An observational audit of 65535 patients in a national dataset. Anaesthesia 2014;69:224-30.

24 Schneeweiss S, Seeger JD, Landon J, Walker AM. Aprotinin during coronary-artery bypass grafting and risk of death. N Engl J Med 2008;358:771-83.

25 Bateman BT, Bykov K, Choudhry NK, Schneeweiss S, Gagne JJ, Polinski JM, et al. Type of stress ulcer prophylaxis and risk of nosocomial pneumonia in cardiac surgical patients: cohort study. BMJ 2013;347:55416.

26 Lindenauer PK, Pekow P, Wang K, Gutierrez B, Benjamin EM. Lipid-lowering therapy and in-hospital mortality following major noncardiac surgery. JAMA 2004:291:2092-9.

27 Lindenauer PK, Pekow P, Wang K, Mamidi DK, Gutierrez B, Benjamin EM. Perioperative beta-blocker therapy and mortality after major noncardiac surgery. $N$ Engl J Med 2005;353:349-61.

28 International Classification of Diseases, ninth revision, Clinical Modification. US Department of Health and Human Services, 1988

29 Memtsoudis SG, Sun X, Chiu YL, Stundner O, Liu SS, Banerjee S, et al. Perioperative comparative effectiveness of anesthetic technique in orthopedic patients. Anesthesiology 2013;118:1046-58

30 Romano PS, Roos LL, Jollis JG. Adapting a clinical comorbidity index for use with ICD-9-CM administrative data: differing perspectives. J Clin Epidemiol 1993;46:1075-9, discussion 81-90.

31 Kulik A, Brookhart MA, Levin R, Ruel M, Solomon DH, Choudhry NK. Impact of statin use on outcomes after coronary artery bypass graft surgery. Circulation 2008:118:1785-92.

32 Rothman KJ, Greenland S, Lash TL. Modern epidemiology. 3rd ed. Wolters Kluwer Health/Lippincott Williams \& Wilkins, 2008.

Accepted: 11 June 2014

\section{Cite this as: BMJ 2014;348:g4022}

This is an Open Access article distributed in accordance with the Creative Commons Attribution Non Commercial (CC BY-NC 3.0) license, which permits others to distribute, 


\section{What is already known on this topic}

Hip fractures are common and serious injuries characterized by high morbidity and mortality risk; surgical repair is generally indicated Few interventions have been shown to decrease mortality in patients with hip fracture

The use of regional anesthesia has been hypothesized to reduce the risk of postoperative mortality among patients undergoing hip fracture surgery, but findings remain conflicting

\section{What this study adds}

In this study of a large nationwide sample of inpatient admissions in the United States, mortality risk did not differ significantly by anesthesia type among patients undergoing surgical repair of hip fracture

If the previously posited beneficial effect of regional anesthesia on postoperative mortality exists, it is likely to be more modest than previously reported 


\section{Tables}

Table 1 | Selected baseline characteristics of study patients receiving general, regional, or combined general and regional anesthesia. Values are numbers (percentages) unless stated otherwise

\begin{tabular}{|c|c|c|c|}
\hline \multirow[b]{2}{*}{ Characteristics } & \multicolumn{3}{|c|}{ Anesthesia type } \\
\hline & General $(n=61554)$ & Regional $(n=6939)$ & General and regional $(n=4791)$ \\
\hline \multicolumn{4}{|l|}{ Personal characteristics } \\
\hline Median (interquartile range) age (years) & $82(74-88)$ & $83(77-88)$ & $83(76-88)$ \\
\hline \multicolumn{4}{|l|}{ Age groups (years): } \\
\hline $18-54$ & $2235(3.6)$ & $99(1.4)$ & $88(1.8)$ \\
\hline $55-69$ & $8224(13.4)$ & $681(9.8)$ & $531(11.1)$ \\
\hline $70-79$ & $13941(22.6)$ & $1581(22.8)$ & $1107(23.1)$ \\
\hline $80-84$ & $12830(20.8)$ & $1540(22.2)$ & $1026(21.4)$ \\
\hline$\geq 85$ & $24324(39.5)$ & $3038(43.8)$ & $2039(42.6)$ \\
\hline Women & $43527(70.7)$ & $5063(73.0)$ & $3429(71.6)$ \\
\hline \multicolumn{4}{|l|}{ Ethnicity: } \\
\hline White & 48768 (79.2) & $5068(73.0)$ & $4084(85.2)$ \\
\hline Black & $2949(4.8)$ & $211(3.0)$ & $247(5.2)$ \\
\hline Hispanic & $1938(3.1)$ & $594(8.6)$ & $39(0.8)$ \\
\hline Other or unknown & $7899(12.8)$ & $1066(15.4)$ & $421(8.8)$ \\
\hline \multicolumn{4}{|l|}{ Hospitalization and surgery characteristics } \\
\hline \multicolumn{4}{|l|}{ Type of admission: } \\
\hline Emergency room & $34852(56.6)$ & $4322(62.3)$ & $2676(55.9)$ \\
\hline Non-healthcare facility & $18554(30.1)$ & $1817(26.2)$ & $1342(28.0)$ \\
\hline Other $^{*}$ & $8148(13.3)$ & $800(11.5)$ & $773(16.1)$ \\
\hline $\begin{array}{l}\text { Median (interquartile range) days before } \\
\text { surgery }\end{array}$ & $1(1-2)$ & $1(1-2)$ & $1(1-2)$ \\
\hline \multicolumn{4}{|l|}{ Type of surgical procedure: } \\
\hline Closed reduction of fracture & $14359(23.3)$ & $1483(21.4)$ & $1047(21.9)$ \\
\hline Open reduction of fracture & $21541(35.0)$ & $2398(34.6)$ & $1470(30.7)$ \\
\hline Total hip replacement & $2622(4.3)$ & $297(4.3)$ & $232(4.8)$ \\
\hline Partial hip replacement & $23252(37.8)$ & $2790(40.2)$ & $2067(43.1)$ \\
\hline Other hip procedure & $3598(5.9)$ & $362(5.2)$ & $336(7.0)$ \\
\hline \multicolumn{4}{|l|}{ Comorbidities } \\
\hline \multicolumn{4}{|l|}{ Type of fracture: } \\
\hline Transcervical fracture & $15951(25.9)$ & $1974(28.4)$ & $1332(27.8)$ \\
\hline Intertrochanteric fracture & $26874(43.7)$ & $2988(43.1)$ & $1941(40.5)$ \\
\hline Subtrochanteric fracture & $3831(6.2)$ & $365(5.3)$ & $243(5.1)$ \\
\hline Trochanteric fracture & $1299(2.1)$ & $152(2.2)$ & $106(2.2)$ \\
\hline Unspecified part of neck of femur & $23754(38.6)$ & $2680(38.6)$ & $1978(41.3)$ \\
\hline Disease related fracture of neck of femur & $1813(2.9)$ & $168(2.4)$ & $111(2.3)$ \\
\hline $\begin{array}{l}\text { Median (interquartile range) Charlson } \\
\text { comorbidity score }\end{array}$ & $1(0-2)$ & $1(0-2)$ & $1(0-2)$ \\
\hline Chronic hypertension & $44521(72.3)$ & $5014(72.3)$ & $3499(73.0)$ \\
\hline Diabetes & $14877(24.2)$ & $1508(21.7)$ & $1049(21.9)$ \\
\hline Previous myocardial infarction & $4966(8.1)$ & $568(8.2)$ & $378(7.9)$ \\
\hline Chronic heart failure & $11020(17.9)$ & $1166(16.8)$ & $838(17.5)$ \\
\hline Chronic obstructive pulmonary disease & $11216(18.2)$ & $1577(22.7)$ & $1070(22.3)$ \\
\hline Chronic renal disease & $10513(17.1)$ & $1023(14.7)$ & $833(17.4)$ \\
\hline Dementia & $17254(28.0)$ & $2081(30.0)$ & $1459(30.5)$ \\
\hline Cancer & $4191(6.8)$ & $405(5.8)$ & $303(6.3)$ \\
\hline
\end{tabular}


Table 1 (continued)

\begin{tabular}{|c|c|c|c|}
\hline \multirow[b]{2}{*}{ Characteristics } & \multicolumn{3}{|c|}{ Anesthesia type } \\
\hline & General $(n=61554)$ & Regional ( $n=6939$ ) & General and regional $(n=4791)$ \\
\hline \multicolumn{4}{|c|}{ Inpatient use of drugs and services before surgery } \\
\hline Angiotensin converting enzyme inhibitor & $8937(14.5)$ & $933(13.4)$ & $673(14.0)$ \\
\hline$\beta$ blocker & $21362(34.7)$ & $2258(32.5)$ & $1582(33.0)$ \\
\hline Calcium channel blocker & $9113(14.8)$ & $1020(14.7)$ & $710(14.8)$ \\
\hline Nitrates & $2391(3.9)$ & $254(3.7)$ & $175(3.7)$ \\
\hline Loop diuretics & $8532(13.9)$ & $878(12.7)$ & $676(14.1)$ \\
\hline Antidiabetes drugs & $9043(14.7)$ & $919(13.2)$ & $664(13.9)$ \\
\hline Statin & $13526(22.0)$ & $1285(18.5)$ & $974(20.3)$ \\
\hline Aspirin & $4166(6.8)$ & $393(5.7)$ & $355(7.4)$ \\
\hline Warfarin & $374(0.6)$ & $25(0.4)$ & $26(0.5)$ \\
\hline Intravenous heparin & $900(1.5)$ & $81(1.2)$ & $61(1.3)$ \\
\hline Intensive care unit admission & $4767(7.7)$ & $432(6.2)$ & $259(5.4)$ \\
\hline Oxygen use & $12568(20.4)$ & $1741(25.1)$ & $1267(26.4)$ \\
\hline Dialysis & $1254(2.0)$ & $104(1.5)$ & $68(1.4)$ \\
\hline \multicolumn{4}{|l|}{ Packed red blood cells transfusion: } \\
\hline 0 unit & $55024(89.4)$ & $6393(92.1)$ & $4290(89.5)$ \\
\hline 1 unit & $2010(3.3)$ & $202(2.9)$ & $139(2.9)$ \\
\hline$\geq 2$ units & $4520(7.3)$ & $344(5.0)$ & $362(7.6)$ \\
\hline \multicolumn{4}{|l|}{ Hospital characteristics } \\
\hline Teaching hospital & $22114(35.9)$ & $1318(19.0)$ & $1273(26.6)$ \\
\hline Urban hospital & $54790(89.0)$ & 5805 (83.7) & 3650 (76.2) \\
\hline
\end{tabular}

Age and Charlson comorbidity score were modeled as categorical variables in the multivariable logistic models. Additional covariates that were included in the multivariable logistic models include marital status; year of admission; hyperlipidemia; history of percutaneous coronary procedure; other forms of ischemic heart disease; chronic cardiac dysrhythmia; previous stroke; liver disease; chronic hemostatic disorders; osteoarthritis; rheumatoid arthritis; obesity; smoking (former or current); alcohol or drug misuse or dependence; history of peptic ulcer disease; unintentional fall; angiotensin receptor blockers, thiazide diuretics; aldosterone antagonists; digoxin; rhythm control drugs; treatment for chronic obstructive respiratory diseases (inhaled $\beta 2$ agonists, inhaled steroids, leukotriene inhibitors, ipratropium, or theophylline); other antiplatelets; subcutaneous anticoagulants; systemic corticosteroids; telemetry; echocardiography; fresh frozen plasma transfusion; thirds of hip fracture surgery volume; and region.

*Includes admission from clinic; admission from court or law enforcement; transfer from ambulatory surgery center; transfer from another home health agency; transfer from same hospital, separate claim; transfer from other healthcare facility; transfer from hospice; transfer from skilled nursing facility or intermediate care facility; transfer from a hospital (different facility); information not available. 
Table 2| Number of events and risk of in-hospital mortality in patients receiving different types of anesthesia. Values are risk ratios (95\% confidence intervals) unless stated otherwise

\begin{tabular}{lccc} 
Variables & General anesthesia & Regional anesthesia & General and regional anesthesia \\
No of patients & 61554 & 6939 & 4791 \\
\hline No of in-hospital deaths & 1362 & 144 & 115 \\
\hline Risk of in-hospital death (\%) & 2.2 & 2.1 & 2.4 \\
\hline Unadjusted analysis & Ref & $0.94(0.79$ to 1.11) & $1.09(0.90$ to 1.32) \\
\hline Adjusted analysis* ${ }^{*}$ & Ref & $0.93(0.78$ to 1.11) & $1.04(0.85$ to 1.26) \\
\hline Fully adjusted analysis† & Ref & $0.93(0.78$ to 1.11) & $1.00(0.82$ to 1.22) \\
\hline Mixed effects analysis $\ddagger$ & Ref & $0.91(0.75$ to 1.10) & $0.98(0.79$ to 1.21)
\end{tabular}

*Adjusted for age, sex, ethnicity, and calendar year. †Included all covariates noted in table 1.

¥Hospital identifying variable was modeled as a normally distributed random intercept, and patient and hospital level characteristics were modeled as fixed effects variables. 
Table 3| Sensitivity analyses: number of events and risk of in-hospital mortality in patients receiving different types of anesthesia. Values are risk ratios $(95 \%$ confidence intervals) unless stated otherwise

Variables

General anesthesia

Regional anesthesia General and regional anesthesia

Patients undergoing hip fracture surgery independently of surgical procedure timing:

\begin{tabular}{|c|c|c|c|}
\hline No of patients & 79915 & 9079 & 6843 \\
\hline No of in-hospital deaths & 1607 & 167 & 142 \\
\hline Risk of in-hospital death (\%) & 2.0 & 1.8 & 2.1 \\
\hline Unadjusted analysis & Ref & $0.91(0.78$ to 1.07$)$ & $1.03(0.87$ to 1.23$)$ \\
\hline Adjusted analysis* & Ref & 0.89 (0.76 to 1.05$)$ & $0.98(0.82$ to 1.16$)$ \\
\hline Fully adjusted analysis $\dagger$ & Ref & $0.91(0.77$ to 1.08$)$ & $0.97(0.82$ to 1.17$)$ \\
\hline \multicolumn{4}{|c|}{ Patients with no recorded diagnosis of cancer at discharge: } \\
\hline No of patients & 49648 & 5565 & 3856 \\
\hline No of in-hospital deaths & 1035 & 110 & 86 \\
\hline Risk of in-hospital death (\%) & 2.1 & 2.0 & 2.2 \\
\hline Unadjusted analysis & Ref & 0.95 (0.78 to 1.16$)$ & $1.07(0.86$ to 1.34$)$ \\
\hline Adjusted analysis* & Ref & $0.94(0.77$ to 1.14$)$ & $1.01(0.81$ to 1.27$)$ \\
\hline Fully adjusted analysis & Ref & $0.93(0.76$ to 1.15$)$ & $0.97(0.77$ to 1.22$)$ \\
\hline \multicolumn{4}{|l|}{ Patients aged 75 years and older: } \\
\hline No of patients & 45709 & 5564 & 3753 \\
\hline No of in-hospital deaths & 1154 & 126 & 96 \\
\hline Risk of in-hospital death (\%) & 2.5 & 2.3 & 2.6 \\
\hline Unadjusted analysis & Ref & $0.89(0.74$ to 1.08$)$ & $1.01(0.82$ to 1.25$)$ \\
\hline Adjusted analysis* & Ref & $0.94(0.78$ to 1.13$)$ & $0.99(0.80$ to 1.22$)$ \\
\hline Fully adjusted analysis & Ref & $0.92(0.76$ to 1.12$)$ & 0.95 (0.77 to 1.18$)$ \\
\hline
\end{tabular}

*Adjusted for age, sex, ethnicity, and calendar year.

†Includes all covariates noted in table 1 except for inpatient use of drugs and services before day of surgery. 


\section{Figure}

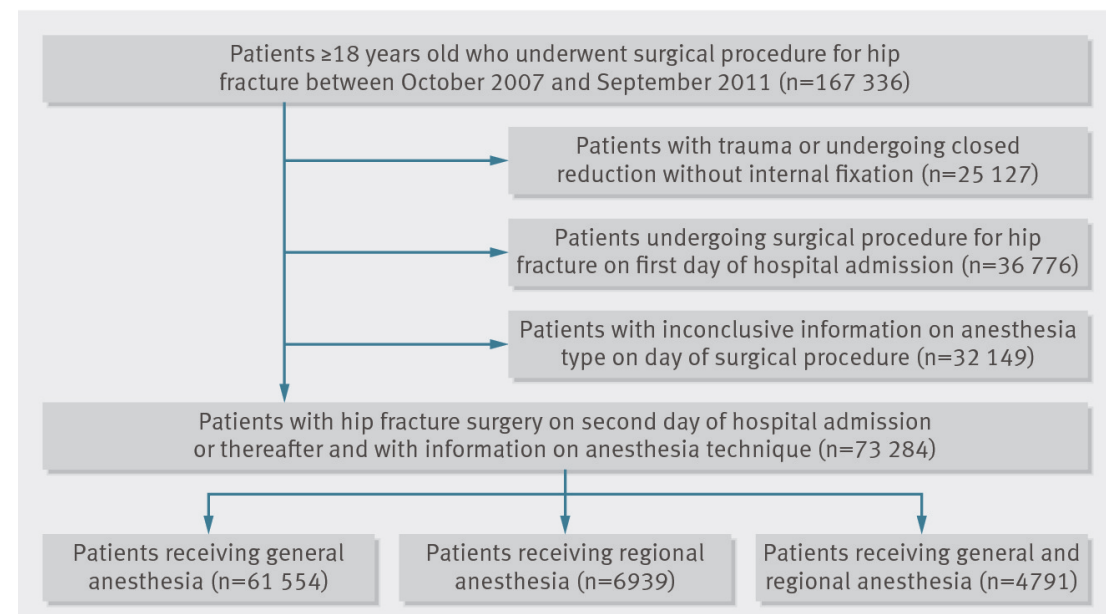

Flowchart of study cohort 